\title{
Dynamics of Structural-Inhomogeneous Laminate and Shell Mechanical Systems with Point Constraints and Focused Masses. Part 2. Statement of the Problem of Forced Oscillations, Methods of Solution, Computational Algorithm and Numerical Results
}

\author{
Mirsaidov Mirziyod', Safarov Ismoil Ibrokhimovich'2, Teshaev Mukhsin Khudoyberdievich ${ }^{3}$ \\ ${ }^{1}$ Tashkent Institute of Irrigation and Agricultural Mechanization Engineers, Tashkent, Uzbekistan \\ ${ }^{2}$ Tashkent Chemical Engineering Institute, Tashkent, Uzbekistan \\ ${ }^{3}$ Bukhara Engineering and Technology Institute, Bukhara, Uzbekistan \\ Email: muhsin_5@mail.ru
}

How to cite this paper: Mirziyod, M., Ibrokhimovich, S.I. and Khudoyberdievich, T.M. (2019) Dynamics of Structural-Inhomogeneous Laminate and Shell Mechanical Systems with Point Constraints and Focused Masses. Part 2. Statement of the Problem of Forced Oscillations, Methods of Solution, Computational Algorithm and Numerical Results. Journal of Applied Mathematics and Physics, 7, 2671-2684. https://doi.org/10.4236/jamp.2019.711182

Received: September 28, 2019

Accepted: November 2, 2019

Published: November 5, 2019

Copyright () 2019 by author(s) and Scientific Research Publishing Inc. This work is licensed under the Creative Commons Attribution International License (CC BY 4.0).

http://creativecommons.org/licenses/by/4.0/ (c) (i) Open Access

\begin{abstract}
A vibrational formulation, a technique, and an algorithm are proposed for assessing the resonance state of a package of rectangular plates and shells having point bonds and concentrated masses with different rheological properties of deformable elements under the influence of harmonic influences. The viscoelastic properties of elements are described using the linear Boltzmann-Volterra theory. An algebraic system of equations with complex coefficients is obtained, which is solved by the Gauss method. Various problems on steady-state forced vibrations for structurally inhomogeneous mechanical systems consisting of a package of plate and shell systems with concentrated masses and shock absorbers installed in it were solved. A number of new mechanical effects have been discovered associated with a decrease in the maximum resonance amplitudes of the mechanical system as a whole. The concept of "global resonance amplitude" is introduced to study the behavior of the resonance amplitudes of a mechanical system. An analysis of the numerical results showed that the interaction of resonant amplitudes is observed only in structurally inhomogeneous systems (in this case, with elastic and viscoelastic elements) and with a noticeable approximation of the natural frequencies.
\end{abstract}




\section{Keywords}

Viscoelastic Shell, Plate, Point Bonds, Vibrational Equation,

Global Resonance Amplitude, Concentrated Mass, Shock Absorber

\section{Introduction}

Mechanical systems, consisting of a package of rectangular plates and shells with point bonds and concentrated masses with various rheological properties of deformable elements under the influence of harmonic influences, are widely used in aircraft manufacturing, mechanical engineering, shipbuilding and other industries and construction. Therefore, great importance is given to the study of forced vibrations of mechanical systems having point bonds and concentrated masses with various rheological properties of deformable elements.

Here are some of these works, for example: In [1] [2], longitudinal and torsional vibrations of a cylindrical shell with concentrated masses at its ends were studied. The work [3] [4] [5] is devoted to the calculation of the forced vibrations of shells of revolution and plates with locally attached bodies. In [6] [7] [8], development trends of the construction industry, aerospace engineering, shipbuilding, chemical and energy industries, and many other branches of modern technology are discussed, which are characterized by the increasing complexity of design decisions in the design of various objects and, in particular, are usually represented by thin-walled spatial structures and on the one hand, they raise the requirements for the reliability of these facilities in operation, and on the other hand, to reduce its weight and material consumption. Also, when designing complex structures, along with traditional metal materials, polymer materials and composites based on them are increasingly used [9] [10] [11]. Modern engineering structures made of composite materials consist of a set of elastic and viscoelastic damping elements with various rheological properties, various types of supports and forces, elastic and viscoelastic constraints with significantly different rheology. Such mechanical systems were classified in works [12] [13] as structurally heterogeneous and the task of developing reliable methods and their analysis has not yet lost its relevance.

The request for practice requires the creation of more accurate methods for solving such problems, and engineering and research organizations and higher educational institutions should focus on computer technologies widely implemented in design. These methods and the algorithms based on them should take into account the actual conditions, structures, behavior as much as possible, carry our calculations without exceeding geometric idealization, consider the real rheological properties of structural elements, the most approximate design schemes to full-scale design and, as a result of the calculations, make a scientifically sound selection of its parameters [14] [15]. Carrying out numerical experiments for complex structurally heterogeneous structures makes it possible to 
understand the qualitative picture of the influence of various parameters and provide reasonable recommendations, not only at the design stage, but also for a model and full-scale experiment, to significantly reduce the amount of experimental research. The development and implementation of the finite element method make it possible to solve the problem of creating a universal program for solving the statics and dynamics of an arbitrary type problem and target structures [16] [17]. The existence of such programs and software systems for a mechanical system consisting of a package of rectangular plates and shells with point bonds and concentrated masses with various rheological properties of deformable elements under the influence of harmonic influences will make judgments on publications. To analyze such structures, it is more efficient to use a model with a discrete continuum [18].

In [19], a new version of the differential-quadrature method was proposed for obtaining the vibrational characteristics of rectangular plates resting on elastic bases and bearing any number of spring-loaded masses. The accuracy of the technique is demonstrated by comparing the calculated results with published data. This work uses an uneven grid distance. The results will also demonstrate the effectiveness of the method in solving the problem of vibration of rectangular plates, bearing any number of sprung masses and resting on elastic bases.

In [20], a low computational cost developed a technique based on the eigenfunction decomposition method for studying the vibration of rectangular plates with a series of moving sprung masses. It is shown that the proposed method can significantly increase the computational efficiency of traditional methods by eliminating a large number of time-varying components in related matrices of ordinary differential equations. The dynamic behavior of the system is then investigated by conducting a comprehensive parametric study of the dynamic amplification of the coefficient of moving loads. The results show that ignoring the flexibility of the vehicle suspension system, both in the model with moving force and in the model with moving mass, can lead to a significant underestimation of the dynamic gain of the coefficient of moving loads.

These methods are effective when the mass is attached by an elastic element and has one degree of freedom. Using the method leads to insurmountable difficulties when considering a package of plates with concentrated masses and discontinuous boundary conditions.

Currently, methods, algorithms and programs for solving the problems of statics and dynamics of mechanical systems of multilayer rectangular plates and shells of elastic material [21] [22], under the influence of dynamic loads, have been sufficiently developed. Further development of plate and shell mechanical systems involves taking into account the rheological properties of the material under vibrational influences [23] [24] [25] [26]. In these works, the forced vibrations of linear viscoelastic mechanical systems under vibrational stress are mainly considered.

The brief review of the works shows that the study of the dynamic behavior of plate mechanical systems with attached masses and various discontinuous boun- 
dary conditions under the influence of external loads is evaluated differently, and each theory or method used has its advantages and disadvantages.

To date, the question of assessing the level of vibrational processes of plate and shell dissipative mechanical systems with attached masses with different boundary conditions remains insufficiently studied and requires extensive study. Therefore, this problem is urgent, requiring a phased solution in the field of protection of electronic equipment from dynamic loads.

\section{The Mathematical Formulation of the Problem of Forced Steady-State Oscillations of Viscoelastic Systems with Point Bonds}

Consider a mechanical system consisting of $N$ isotropic viscoelastic bodies (a package of rectangular plates or cylindrical shells) that occupy a volume $V_{n}$ and are limited by surfaces $\Omega_{n}(n=1,2, \cdots, N)$. It is assumed that one linear size of each body is much smaller than the other two (class of plates and shells).

For each $n$, homogeneous boundary conditions are set on a part of the surface $\Omega_{n}^{f r}=\Omega_{n} / \Omega_{n}^{b o}$ of the $n$-the body $\Omega_{n}^{b o}$, kinematic and (or) dynamic relationships are imposed on the remaining free surface at a finite number of points: point rigid, elastic and (or) viscoelastic articulated type supports (rigid supports can be pinched), rigid elastic and (or) viscoelastic shock absorbers (massless viscoelastic elements), connecting bodies (at $N>1$ ), concentrated masses $M_{q n}(q=1,2, \cdots, Q)$. The arrangement of bonds and masses on surfaces $\Omega_{n}^{f r}$ is arbitrary. At the edges of the plates or shells, uniform boundary conditions are specified.

In the particular case, such a structurally inhomogeneous viscoelastic system consists of a finite number $(N)$ of elastic and (or) viscoelastic with massive and without mass elements.

The forced oscillations of a structurally inhomogeneous viscoelastic mechanical system that occur in the presence of external periodic influences are considered. The study of this type of oscillation of the system allows us to identify the dependence of the maximum amplitudes of displacements and stresses at any point in the system on the parameters of the system itself and external influences. In this case, the dissipative properties of the system are manifested mainly in resonance modes. The values of the resonance amplitudes of displacements and stresses are used as a quantitative assessment of the intensity of dissipative processes. It is required to determine the amplitude-frequency characteristics of several characteristic points of a structurally inhomogeneous mechanical system, on the basis of these construct a graph of the change in the resonance amplitude of the mechanical system depending on the geometric and physic-mechanical parameters of the system [23].

Suppose that external forces applied to the nth body have different amplitudes, but the same frequencies; then the law of their change can be written as

$$
\bar{P}_{n j}(t)=\bar{P}_{n j}^{0} \mathrm{e}^{-i \Omega t} \quad(n=1, \cdots, N ; j=1, \cdots, J)
$$


where $\Omega$ is the set actual frequency of the external disturbing force; $J$ is the number of components of the displacement vector; $\bar{P}_{n j}^{0}$ is the vector of amplitudes of the external disturbing force directed along the $j$-the component of the displacement vector $P_{n j}(\bar{x}, t) ; N$ is the number of elements in the system. To describe the relaxation processes occurring in point bonds or viscoelastic elements of the system, we accept the linear hereditary Boltzmann-Volterra theory:

$$
\sigma_{m k}^{n}(t)=E_{n}\left[+\varepsilon_{m k}^{n}(t)-\int_{-\infty}^{t} R^{n}(t-\tau) \cdot \varepsilon_{m k}^{n}(\tau) \mathrm{d} \tau\right],
$$

where $R^{n}(t)$ is the relaxation core of the $n$-the viscoelastic element or point bond, $E_{n}$ is the instantaneous elastic modulus.

In order for the stress to be a periodic function of time in the expression of heredity (2), the lower limit of the integral must be equal to minus infinity. The voltage will contain an aperiodic component if the lower limit is zero.

Here, in contrast to the problems of natural vibrations, the smallness of the parameters of the relaxation core is not assumed. Poisson's ratio is assumed constant ( $v=$ const). If $R(t)=0$, then the system is elastic.

We use the principle of possible (virtual) movements, which is realized by an equation similar to (2), with an additional term-the virtual work of surface forces $\delta A_{p}$ :

$$
\delta A_{a}+\delta A_{m}+\delta A_{p}+\delta A_{\sigma}=0,
$$

where $\delta A_{\sigma}, \delta A_{I}, \delta A_{m}$-the virtual work of the internal forces of the bodies of the springs, as well as the forces of inertia, taking into account the concentrated masses. These works can be represented by the following relationships:

$$
\begin{gathered}
\delta A_{a}=-\sum_{n=1}^{N-1} \sum_{l=1}^{L_{n}} \sigma_{l}^{n} \delta \varepsilon_{l}^{n}-\sum_{n=1}^{N} \sum_{l^{\prime}=1}^{L_{n}^{\prime}} \sigma_{l^{\prime}}^{n} \delta \varepsilon_{l^{\prime}}^{n} \\
\delta A_{m}=-\sum_{n=1}^{N} \rho_{n} \int\left(\sum_{V_{n}}^{J} \ddot{U}_{n j}(\bar{x}, t) \delta U_{n j}\right) \mathrm{d} V-\sum_{n=1}^{N} \sum_{q=1}^{Q} M_{q n} \sum_{j=1}^{J} \ddot{U}_{n j}\left(x_{n 1}^{q}, t\right) \delta U_{n j} \\
\delta A_{p}=\sum_{n=1}^{N} \sum_{j=1}^{J} \bar{P}_{n j}(t) \int_{S_{n}} \delta U_{n j}(\bar{x}, t) \mathrm{d} S \\
\delta A_{\sigma}=-\sum_{n=1}^{N} \sigma_{m k}^{n} \delta \varepsilon_{m k}^{n} \mathrm{~d} V
\end{gathered}
$$

where $\bar{x}_{n}^{q}=\left(\bar{x}_{n 1}^{q}, \bar{x}_{n 2}^{q}, \bar{x}_{n 3}^{q}\right)$ are the coordinates, $L_{n}$ is the number of deformable elements (springs or shock absorbers) between the $n$ and the $(n+1)$-bodies, $Q_{n}$ is the number of concentrated masses on the n-the body, $L_{n}^{\prime}$ is the number of elastic (viscoelastic) supports on the $n$-the body, $\sigma_{m k}^{n}, \varepsilon_{m k}^{n}, \sigma_{l}^{n}, \varepsilon_{l}^{n}, \sigma_{l^{\prime}}^{n}, \varepsilon_{l^{\prime}}^{n}$ are the components of the stress and strain tensors of the $n$-the body, the $l$-the spring (deformable element or shock absorber) and $l^{\prime}$-the elastic (viscoelastic) support, respectively, $U_{n j}$-the $n$-the component of the displacement vector, $S_{n}$ is the area of the $n$-th element.

The law of steady-state oscillations of the nth element of system (4) will be sought in the form 


$$
U_{n j}(\bar{x}, t)=U_{n j}^{0}(\bar{x}) \mathrm{e}^{-i \Omega t},
$$

where $U_{n j}^{0}(\bar{x})$ is the complex amplitude of the forced oscillations. In contrast to the problem of natural oscillations, here $\Omega$ the frequency will be real.

We transform the variational Equation (4) in the same way as in [1], substituting relations (3), (4), (5) into it, and expressing the strains through the components of the displacement vector $U_{n j}(\bar{x}, t)$. We transform $t-\tau=z$ the integral terms of the type (2) contained in the equation by changing the variable to the form

$$
\int_{-\infty}^{t} R(t-\tau) \varphi(\tau) \mathrm{d} \tau=\left[i \Gamma_{s}(\Omega)+\Gamma_{c}(\Omega)\right] \varphi(t) .
$$

where $\Gamma_{c}(\Omega), \Gamma_{s}(\Omega)$ are relations that differ from the cosine and sine of the Fourier transforms only by numerical factors. This will eliminate the time function $\varphi(t)$ (which in this case has the form $\varphi(t)=\mathrm{e}^{-i \Omega t}$ ) and obtain a variation equation for the displacement vector.

Point connections, as in the problem of natural vibrations, are introduced under the sign of variation using the method of Lagrange multipliers. Then the variational equation will have the form:

$$
\delta\left\{G\left(F\left(\lambda_{n j}^{s}, k_{n j}^{s}, \mu_{n j}^{s}\right)+L\left(U_{n j}^{0}(\bar{x}), \Omega^{2}\right)\right)\right\}=0,
$$

where $L$ - is the total virtual work of the mechanical system, and $F$ - are the kinematic conditions of rigid point connections superimposed on the system.

The task is now formulated as follows: let $\bar{P}_{n j}(t)$-the forcing external force satisfy relations (1); depending on the frequency $\Omega$ of the driving external force, it is required to find the modulus of the displacement vector (amplitude of the forced oscillations) $U_{n j}^{0}(\bar{x})$ satisfying the given homogeneous boundary conditions and equation (6). The Lagrange multipliers (if necessary) can also be determined, the physical meaning of which is the reaction of point rigid bonds.

\section{Construction of Solving Equations of Linear Problems on Forced Steady-State Oscillations of Dissipative Mechanical Systems}

The solution to variational Equation (6) will be sought in the form of a superposition of fundamental basic orthogonal functions. For elements free of concentrated masses, and all point connections (supports, racks), they are assumed to be known. Then, as the desired displacement field satisfying the given homogeneous boundary conditions and the variational Equation (6), we take a finite sum of these fundamental basic orthogonal functions [24]:

$$
U_{n j}^{0}(x)=\sum_{k=1}^{K} \gamma_{n j}^{k} \Phi_{n j}^{k}(\bar{x}), \quad(n=1, \cdots, N ; j=1, \cdots, J)
$$

where $\gamma_{n j}^{k}$ are the unknown coefficients.

In the equations obtained after substituting the sum (7) into relation (6), the Lagrange coefficients $\lambda_{n j}^{s}, k_{n j}^{s}, \mu_{n j}^{r}$ and $\gamma_{n j}^{k}$ factors will be generalized coordinates. 
The dimension of the system will be the same as in the case of free oscillations. It can be represented in matrix form as

$$
\left(A+\sum_{n=1}^{N-1} f_{l n}(\Omega) A_{l n}^{n}+\sum_{n=1}^{N_{n}} f_{n}(\Omega) A_{n}^{n}+\sum_{n=1}^{N} \sum_{l^{\prime}=1}^{L^{\prime}} f_{l^{\prime} n}(\Omega) A_{l^{\prime} n}^{n}-\Omega^{2} B\right) \bar{\xi}=\hat{P}_{n j}^{0} .
$$

A vector-a column $\hat{P}_{n j}^{0}$-structurally consists of two subvectors. If equation (8) is first differentiated with respect to the Lagrange multipliers $\lambda_{n j}^{s}, k_{n j}^{s}, \mu_{n j}^{r}$, then the upper subvector of the vector $\vec{P}^{0}$-becomes zero, and its dimension will be equal to the total number of Lagrange multipliers. The vector of amplitudes of the driving forces will be the lower subvector $\vec{P}_{n j}^{0}$. Moreover, in formula (1) it is assumed that the external perturbing force has a distributed character. The task, in fact, will not change if the applied force is taken concentrated. Then the virtual work on the area of the element will be replaced by the virtual work of the concentrated force, and only the amplitude vector $\vec{P}_{n j}^{0}$ will change, which, in addition to the amplitude of the concentrated force, will be filled with zeros.

We solve the system (8) by the Gauss method with the selection of the main element in columns and also in rows. The initial system of Equations (8) has complex coefficients; therefore, a program that implements the algorithm was written for systems with complex coefficients and complex unknowns.

The right side of the system, i.e. vector $\hat{P}_{n j}^{0}$, set in the form

$$
\vec{P}_{n j}^{0}=i \cdot \overline{I P}_{n j}^{0}+\bar{R} \bar{P}_{n j}^{0},
$$

where $\bar{R} \bar{P}_{n j}^{0}, \overline{I P}_{n j}^{0}$ are the real and imaginary parts of the load vector, moreover,

$$
\overline{I P}_{n j}^{0}=0,
$$

The components of the vector of unknowns will be complex quantities, i.e. $\bar{\xi}^{\prime}$-the vector can be represented as $\bar{\xi}^{\prime}=i \bar{\xi}_{I}+\bar{\xi}_{R}$. In order for the coupling reactions and the amplitudes of the forced vibrations to make real sense, we must accept: $\bar{\xi}^{\prime}=|\bar{\xi}|$, i.e., as the generalized coordinates $\lambda_{n j}^{s}, k_{n j}^{s}, \mu_{n j}^{r}, \gamma_{n j}^{k}$, we must take the modules of the corresponding components of the vector $\bar{\xi}$. Then the components of the displacement vector can be uniquely determined by $\gamma_{n j}^{k}$ formula (7). Substituting the coordinates of any point in the system into the last formula, we obtain the amplitude of its oscillations for a given driving frequency $\Omega$.

\section{Numerical Implementation of the Algorithm}

In this section, several problems will be solved in which the amplitude-frequency characteristics for the displacements of individual points of structurally inhomogeneous viscoelastic systems will be obtained and analyzed.

Example 1. In this problem, the amplitude-frequency characteristics of mechanical systems are determined depending on its geometric parameters. The mechanical system is a package of two square elastic plates connected in the center by one weightless viscoelastic shock absorber. On both plates there is one attached mass. The plates are articulated on the contour, the same in geometric 
and mechanical parameters:

$$
E=2 \times 10^{11} \mathrm{~N} / \mathrm{m}^{2}, \rho=7.8 \times 10^{3} \mathrm{~kg} / \mathrm{m}^{2}, v=0.35, h=0.001 \mathrm{~m} .
$$

The relaxation core for viscoelastic shock absorbers is selected as the Rzhanitsyn-Koltunov core, i.e.:

$$
R(t)=A \mathrm{e}^{-\beta t} t^{\alpha-1},
$$

where A, $\alpha, \beta$ are the kernel parameters [25]. The viscosity of the shock absorber is adopted such that its creep deformation during the quasistatic process is a small fraction $(\sim 12 \%)$ of the total deformation.

The parameters of the relaxation core (10) are as follows: $A=0.01, \alpha=0.1, \beta=0.05$. This corresponds to approximately $60 \%$ of the contribution of creep strain to the total deformation of a viscoelastic body under a quasistatic loading process. The instantaneous stiffness $C_{0}$ of the shock absorber is fixed and taken equal to 10 .

The masses are equal to each other $\left(M_{1}=M_{2}=0.05 \mathrm{~kg}\right)$, one of them $\left(M_{1}\right)$ $x_{M_{1}}=0.14, y_{M_{1}}=0.1 \mathrm{~m}$ is fixed on the lower plate at a point, and the other $\left(M_{2}\right)$ $x_{M_{2}}=0.02 \mathrm{~m}, y_{M_{2}}=0.1 \mathrm{~m}$ is fixed on the upper plate at a point or. As a harmonically exciting load, a driving harmonic load $P(t)$ uniformly distributed over the area of both plates is considered. The amplitude vector of this load is equal to a unit vector. Depending on the position of the load $M_{2}$, the amplitudes of displacements of the forced vibrations of the central points $(x=y=0.1 \mathrm{~m})$ of the lower and upper plates were found depending on the frequency of the forced loads.

Figure 1 and Figure 2 show the case when the mass $M_{2}$ is at the point of the upper plate $x_{M_{2}}=0.02 \mathrm{~m}, y_{M_{2}}=0.1 \mathrm{~m}$.

At these positions of mass $M_{2}$, the qualitative pictures of the amplitude-frequency characteristics of the upper and lower plate.

It can be seen from Figure 3 and Figure 4 that, at $x_{M_{2}}=0.18 \mathrm{~m}, y_{M_{2}}=0.1 \mathrm{~m}$ coordinates $M_{2}$, the damping ability of the structure is the same.

Thus, the coincidence of the graphs of the amplitude-frequency characteristics at different locations of the mass $M_{2}$ confirms the symmetry of Figure 3 and Figure 4.

Here, two central points on the lower and upper plates are selected as characteristic points. Points with other coordinates give qualitatively the same results.

Example 2. We consider a structurally no uniform system consisting of elastic rectangular plates with concentrated masses, connected in the center by one weightless viscoelastic shock absorber (Figure 5). The concentrated mass is applied at a point $x=y=0.1 \mathrm{~m}$ and $M_{1}=1$.

In this case, in specific calculations, the kernel parameters are as follows:. The coefficient of stiffness moments $C_{0}$ ranged from $10^{-4}$ to $10^{-1}$.

As a harmonically exciting load, a driving harmonic load $P(t)$ uniformly distributed over the area of the upper plate is considered. The amplitude vector of this load is equal to a unit vector. 
One can introduce the concept of "global resonance amplitude" to study the behavior of the resonance amplitude of a mechanical system depending on the geometric and physico-mechanical parameters of mechanical systems: where $\delta_{A}=\max _{k}\left|A_{k}\right|$ is the $k$-number of the resonant peak.

Figure 5 shows a graph of the global coefficient of resonance amplitude $\delta_{A}$ versus the instantaneous stiffness of the $C_{0}$ shock absorber for a structurally inhomogeneous mechanical system of the central point of the bottom plate. Depending on the value of $C_{0}$, the amplitude $\delta_{A}$ is determined either by the first, second, or third resonance peak. The minimum $\delta_{A}$ value corresponds to the value of $C_{0}$ at which the low frequencies (first and second) of the mechanical system get as close as possible.

The role of the global resonance amplitude depending on $C_{0}$ for homogeneous mechanical systems is played by the resonance amplitude corresponding to the first frequency (Figure 5). For dissipative inhomogeneous mechanical systems, the resonance amplitude is corresponding to both the first and second frequencies. "Role change" occurs at the point where the real parts of the natural frequencies are closest to each other (Figure 6).

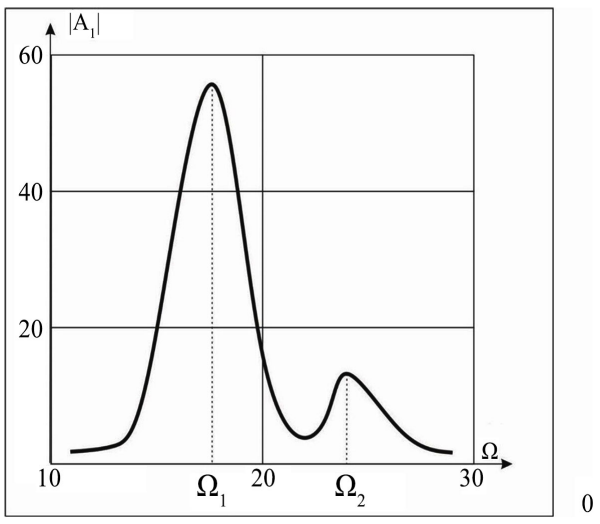

Figure 1. Frequency response plotted for the center points of the bottom plate at $x_{M_{1}}=0.14, y_{M_{1}}=0.1 \mathrm{~m}, x_{M_{2}}=0.02 \mathrm{~m}, y_{M_{2}}=0.1 \mathrm{~m}$.

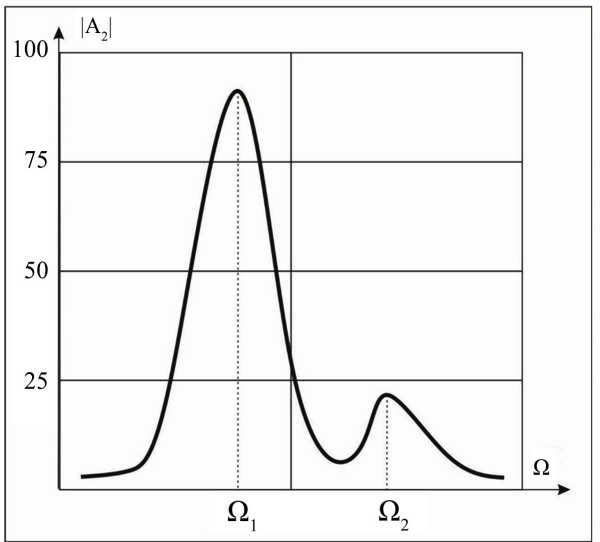

Figure 2. Frequency response constructed for the center points of the upper plate at $x_{M_{1}}=0.14, y_{M_{1}}=0.1 \mathrm{~m}, x_{M_{2}}=0.02 \mathrm{~m}, y_{M_{2}}=0.1 \mathrm{~m}$. 


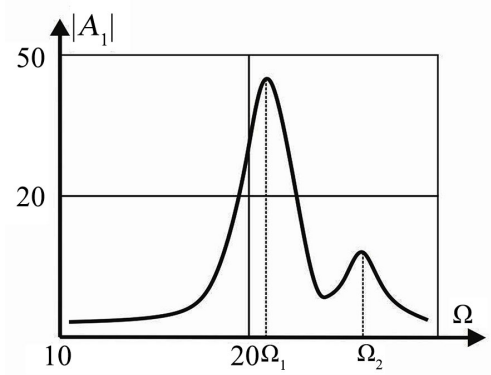

Figure 3. Frequency response plotted for the center points of the bottom plate at $x_{M_{1}}=0.14, y_{M_{1}}=0.1 \mathrm{~m}, x_{M_{2}}=0.18 \mathrm{~m}, y_{M_{2}}=0.1 \mathrm{~m}$.

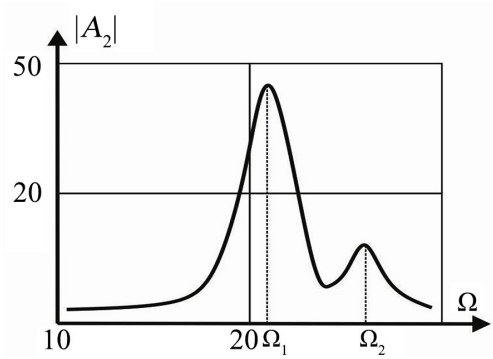

Figure 4. Frequency response constructed for the center points of the upper plate at $x_{M_{1}}=0.14, y_{M_{1}}=0.1 \mathrm{~m}, x_{M_{2}}=0.18 \mathrm{~m}, y_{M_{2}}=0.1 \mathrm{~m}$.

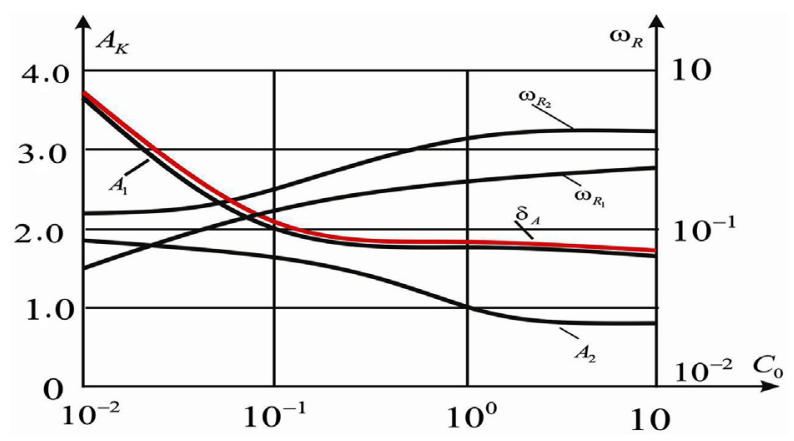

Figure 5. Change in the coefficient of the global resonant amplitude relative to the stiffness of the shock absorber (Structurally homogeneous system).

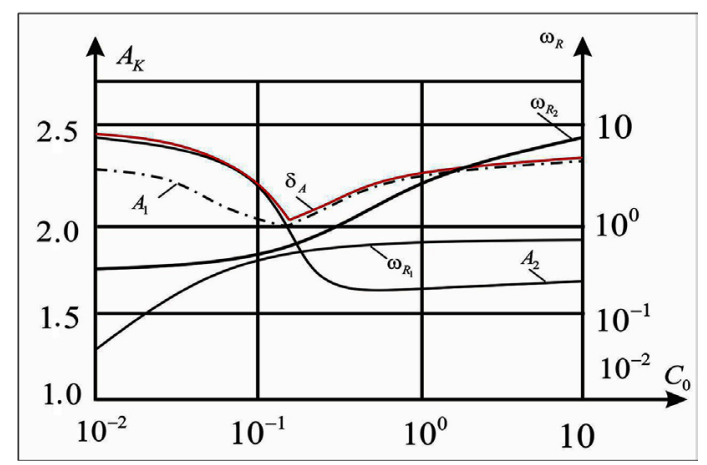

Figure 6. Change in the coefficient of the global resonant amplitude relative to the stiffness of the shock absorber (Structurally inhomogeneous system). 
At this point, i.e. at the point where the "Change of Roles" is observed, the global resonance amplitude $\delta_{A}$ reaches its minimum. At this point, in comparison with other points, the energy intensity will be the highest (intense).

Thus, the results obtained for the structurally inhomogeneous viscoelastic structure under consideration are completely consistent with the solutions of the problem of free damped vibrations and confirm the fact of a sharp increase in the intensity of dissipative processes when the fundamental frequencies approach each other in inhomogeneous viscoelastic systems.

\section{Conclusions}

1) Based on the principle of possible displacements, a mathematical formulation and methods for solving the problem of forced vibrations of structurally homogeneous and inhomogeneous mechanical systems consisting of a package of plates (or shells) with point supports and attached masses under the influence of harmonic loads are formulated.

2) The problems of forced oscillations of dissipative homogeneous and inhomogeneous plate systems with concentrated supports and mass attachments are solved.

3) To describe the dissipative properties of the system as a whole, the concept of "global resonance amplitude" is introduced. In the case of a structurally homogeneous mechanical system, the global resonant amplitude is entirely determined by the resonant amplitude corresponding to the first natural frequency.

4) In the case of a structurally inhomogeneous mechanical system, the global resonance amplitude, depending on the stiffness of the shock absorber, is played by the resonance amplitudes corresponding to the first natural frequency (up to $C=C_{0}$ ), and then the resonant amplitude corresponding to the second natural frequency.

\section{Recommendations}

In some cases, the real structure working under vibrational influences can be represented as a combination of plate dissipatively inhomogeneous mechanical systems with an attached finite number of rigid masses, and others can have a complex structure due to the use of various materials with different rheological properties. Therefore, when designing such structures, it is constantly necessary to solve the problems of calculating the strength of the underlying change in the global resonance amplitude from the geometric and physico-mechanical parameters of the system's elements.

\section{Conflicts of Interest}

The authors declare no conflicts of interest regarding the publication of this paper.

\section{References}

[1] Hsu, M.H. (2003) Vibration Analysis of Isotropic and Orthotropic Plates with Mixed 
Boundary Conditions. Tamkang Journal of Science and Engineering, 6, 217-226.

[2] Hsu, M.H. (2004) Nonlinear Dynamic Analysis of an Orthotropic Composite Rotor Blade. Journal of Marine Science and Technology, 12, 247-255.

[3] Hsu, M.H. (2005) Vibration Analysis of Edge-Cracked Beam on Elastic Foundation with Axial Loading Using the Differential Quadrature Method. Computer Methods in Applied Mechanics and Engineering, 194, 1-17. https://doi.org/10.1016/j.cma.2003.08.011

[4] Muresan, A.-A., Nedelcu, M. and Gonçalves, R. (2019) GBT-Based FE Formulation to Analyze the Buckling Behavior of Isotropic Conical Shells with Circular CrossSection. Thin- Walled Structures, 134, 84-101. https://doi.org/10.1016/j.tws.2018.07.032

[5] Yu, W., Hodges, D. and Ho, J.C. (2012) Variational Asymptotic Beam Sectional Analysis-An Updated Version. International Journal of Engineering Science, 59, 40-64. https://doi.org/10.1016/j.ijengsci.2012.03.006

[6] Gbadeyan, J. and Dada, M. (2011) A Comparison of Dynamic Responses of Three Versions of Moving Load Problem Involving Elastic Rectangular Plates. Journal of Vibration and Control, 17, 903-915. https://doi.org/10.1177/1077546310377910

[7] Ghafoori, E., Kargarnovin, M.H. and Ghahremani, A.R. (2011) Dynamic Responses of a Rectangular Plate under Motion of an Oscillator Using a Semi-Analytical Method. Journal of Vibration and Control, 17, 1310-1324. https://doi.org/10.1177/1077546309358957

[8] Ghazvini, T., Nikkhoo, A., Allahyari, H. and Zalpuli, M. (2016) Dynamic Response Analysis of a Thin Rectangular Plate of Varying Thickness to a Traveling Inertial Load. Journal of the Brazilian Society of Mechanical Sciences and Engineering, 38, 403-411. https://doi.org/10.1007/s40430-015-0409-2

[9] Baeza, L. and Ouyang, H. (2009) Vibration of a Truss Structure Excited by a Moving Oscillator. Journal of Sound and Vibration, 321, 721-734. https://doi.org/10.1016/j.jsv.2008.09.049

[10] Celebi, E. (2006) Three-Dimensional Modeling of Train-Track and Sub-Soil Analysis for Surface Vibrations Due to Moving Loads. Applied Mathematics and Computation, 179, 209-230. https://doi.org/10.1016/j.amc.2005.11.095

[11] Chang, T.P. (2014) Stochastic Dynamic Finite Element Analysis of Bridge-Vehicle System Completed to Random Material Properties and Loadings. Applied Mathematics and Computation, 242, 20-35. https://doi.org/10.1016/j.amc.2014.05.038

[12] Dimitrovová, Z. (2018) Complete Semi-Analytical Solution for a Uniformly Moving Mass on a Beam on a Two-Parameter Visco-Elastic Foundation with Non-Homogeneous Initial Conditions. International Journal of Mechanical Sciences, 144, 283 311. https://doi.org/10.1016/j.ijmecsci.2018.05.055

[13] Eftekhari, S. (2016) A Modified Differential Quadrature Procedure for Numerical Solution of Moving Load Problem. Proceedings of the Institution of Mechanical Engineers Part C, 230, 715-731. https://doi.org/10.1177/0954406215584630

[14] Gupta, U.S., Lal, R. and Sharma, S. (2007) Vibration of Non-Uniform Circular Plates with Variable Thickness. Journal of Sound and Vibration, 302, 1-17. https://doi.org/10.1016/j.jsv.2006.07.005

[15] Huang, M., Ma, X.Q., Sakiyama, T., Matsuda, H. and Morita, C. (2007) Free Vibration Analysis of Rectangular Plates with Variable Thickness and Point Support. Journal of Sound and Vibration, 300, 435-452.

https://doi.org/10.1016/j.jsv.2005.01.059 
[16] Jin, G.Y., Su, Z., Shi, S.X., Ye, T.G. and Gao, S.A. (2014) Three-Dimensional Exact Solution for the Free Vibration of Arbitrarily Thick Functionally Graded Rectangular Plates with General Boundary Conditions. Composite Structures, 108, 565-577. https://doi.org/10.1016/j.compstruct.2013.09.051

[17] Kerboua, Y., Lakis, A.A., Thomas, M. and Marcouiller, L. (2007) Hybrid Method for Vibration Analysis of Rectangular Plates. Nuclear Engineering Design, 237, 791-801. https://doi.org/10.1016/j.nucengdes.2006.09.025

[18] Lal, R. and Dhanpati (2007) Transverse Vibrations of Non-Homogeneous Orthotropic Rectangular Plates of Variable Thickness: A Spline Technique. Journal of Sound and Vibration, 306, 203-214. https://doi.org/10.1016/j.jsv.2007.05.014

[19] Safarov, I.I., Boltaev, Z.I. and Axmedov, M.S. (2015) Setting the Linear Oscillations of Structural Heterogeneity Viscoelastic Lamellar Systems with Point Relations. Applied Mathematics, 6, 228-234. https://doi.org/10.4236/am.2015.62022

[20] Safarov, I.I., Teshaev, M.K. and Majidov, M. (2014) Damping of Oscillations of Mechanical Systems. LAP (LAMBERT Academik Publishing), Latvia.

[21] Koltunov, M.A. (1976) Creep and Relaxation. Higher Cleavage

[22] Safarov, I.I. (1992) Oscillations and Waves in Dissipatively Inhomogeneous Media and Structures. FAN, Tashkent.

[23] Safarov, I.I., Teshayev, M.K. and Axmedov, M.S. (2014) Natural Oscillations of Viscoelastic Lamellar Mechanical Systems with Point Communications. Applied Mathematics, 5, 3018-3025. https://doi.org/10.4236/am.2014.519289

[24] Mirsaidov, M.M. and Sultanov, T.Z. (2013) Use of Linear Heredity Theory of Viscoelasticity for Dynamic Analysis of Earthen Structures. Soil Mechanics and Foundation Engineering, 49, 250-256. https://doi.org/10.1007/s11204-013-9198-8

[25] Koltunov, M.A., Mirsaidov, M.M. and Troyanovskii, I.E. (1978) Transient Vibrations of Axissymmetric Viscoelastic Shells. Polymer Mechanics, 14, 233-238. https://doi.org/10.1007/BF00857468

[26] Mirsaidov, M.M. and Troyanovskii, I.E. (1975) Forced Axisymmetric Oscillations of a Viscoelastic Cylindrical Shell. Polymer Mechanics, 11, 953-955. https://doi.org/10.1007/BF00857626 


\section{Designations}

\section{Notation content}

$N$ : the number of system elements

$V_{n}$ : volume

$\Omega_{n}(n=1,2, \cdots, N)$ : bounded surfaces

$\Omega_{n}^{b o}$ : surface of homogeneous boundary conditions

$M_{q n}(q=1,2, \cdots, Q):$ concentrated masses

$\Omega_{n}^{\text {fr }}$ : Surface arrangement of bonds and masses

$\Omega$ : the actual frequency of external disturbing forces

$J$ : the number of displacement vector components

$\bar{P}_{n j}^{0}$ : vector of amplitudes of external disturbing force

$R^{n}(t)$ : the relaxation core of the nth viscoelastic element

$E_{n}$ : instant modulus of elasticity

v. is the Poisson's ratio

$\delta A_{\sigma}$ : virtual work of internal forces

$\delta A_{I}$ : virtual work of inertia force

$\delta A_{m}$ : virtual work of concentrated masses

$L_{n}$ : the number of deformable elements

$L_{n}^{\prime}$ : the number of viscoelastic supports

$Q_{n}$ : number of concentrated masses on the $n$-th body

$\sigma_{m k}^{n}, \varepsilon_{m k}^{n}, \sigma_{l}^{n}, \varepsilon_{l}^{n}, \sigma_{l^{\prime}}^{n}, \varepsilon_{l^{\prime}}^{n}:$ components of stress and strain tensors $n$-th body

$U_{n j}: n$-th component of the displacement vector

$S_{n}:$ area of the $n$-th element

$U_{n j}^{0}(\bar{x})$ : complex amplitude of forced oscillations

$U_{n j}(\bar{x}, t)$ : components of displacement vector

$\Gamma_{c}(\Omega), \Gamma_{s}(\Omega)$ : relations, from the cosine and sine of the Fourier transforms

$L$ : total virtual work of the mechanical system

$F$ : kinematic conditions of rigid point connections

$\gamma_{n j}^{k}:$ unknown coefficients

$\lambda_{n j}^{s}, k_{n j}^{s}, \mu_{n j}^{r}:$ Lagrange multipliers

A, $\alpha, \beta$ : relaxation core parameters

$\bar{R} \bar{P}_{n j}^{0}, \overline{I P}_{n j}^{0}$ :real and imaginary parts of the load vector

$\bar{\xi}^{\prime}=i \bar{\xi}_{1}+\bar{\xi}_{R}$ : vectors of unknown complex quantities

$M_{1}$ and $M_{2}$ : concentrated masses

$\delta_{A}:$ global resonance amplitude

$C_{0}$ : koefficient instant shock absorber stiffness 